\title{
Which of the five classical ballet positions is the most demanding for the dancer's body? An electromyography-based study to determine muscular activity
}

\author{
JOANNA GORWA*, JAROSŁAW KABACIŃSKI, MiCHAŁ MURAWA, ANNA FRYZOWICZ \\ Department of Biomechanics, Chair of Theory and Methodology of Sport, \\ Poznan University of Physical Education, Poznań, Poland.
}

\begin{abstract}
Purpose: The study aimed to determine which of the five classical ballet positions is the most demanding regarding muscular activity, values of external rotation in the hip joints, angular values of foot progression as well as the inclination (tilt) of the pelvis in the sagittal plane. Methods: In this cross-sectional study, 14 female pre-professional ballet dancers (aged 11-16) participated. Participants were tasked with the sequential adoption of five classical ballet positions (CP1-CP5). The electromyographic activity of the muscles of the trunk and the lower limb was recorded with surface electrodes. Kinematic data including hip and knee external rotation, foot progression angle and pelvic tilt were collected using a motion capture system. Results: Symmetric positions CP1 and CP2 were not as demanding as asymmetric CP3-CP5. Higher values of hip and foot external rotation without greater muscular effort in $\mathrm{CP} 2$ than $\mathrm{CP} 1$ was noticed. Considering asymmetric positions, CP3 did not trigger a greater activity of hip or foot muscular groups than CP4 and CP5. CP4 was characterised by the greatest pelvic anterior tilt and the lowest activity of GM in the forward lower limb. In CP5, forward lower limb entailed a higher activity of muscles supporting the foot than in the remaining positions. Conclusion: In terms of biomechanics, the most demanding classical ballet position in pre-professional dancers is CP4, followed by CP5, CP3, CP1 and CP2. This finding can be applied in educational methodology of dancers, figure skaters, synchronized swimmers, acrobatic gymnasts, rhythmic gymnasts or cheerleaders.
\end{abstract}

Key words: motion capture, electromyography, ballet, muscles, turnout, classical dance

\section{Introduction}

There are five classical ballet positions (Fig. 1). The first technical task (concerning lower limbs) involves the mastering of these positions [22]. In dance, such positions, referred to as les bonnes positions, play an essential "initial" role and, regardless of a style, are treated as the primary base or foundation. Contrary to appearances, the aforesaid positions are not only seen in dancehalls of ballet schools but are also indispensable technical elements in figure skating, syn- chronised swimming, acrobatic gymnastics, rhythmic gymnastics or cheerleading [2], [5]. Dancers of various techniques (contemporary, jazz, folklore, ballroom dance) learn the positions enriching their professional workshop [1].

The foundation of classical ballet positions is Turnout (TO), allowing the foot and the knee (of both lower limbs) to be turned in entirely opposite directions and adopt an angle of $180^{\circ}$. The proper TO is initiated in the hip joints [4], [7], [8], [22]. The mastering of technically correct classical ballet positions requires multiannual training and, obviously, appropriate dancer's

\footnotetext{
* Corresponding author: Joanna Gorwa, Department of Biomechanics, Poznan University of Physical Education, ul. Królowej Jadwigi 27/39, 61-871 Poznań, Poland, Phone: +48 6183553 91, e-mail: gorwa@awf.poznan.pl

Received: May 18th, 2020

Accepted for publication: July 27th, 2020
} 
anatomy and predisposition, defined as the natural and high value of external rotation in hip joints [7], [18], [24].

The most important muscles maintaining TO are deep external hip rotators (piriformis, obturator internus and externus, quadratus femoris, gemellus superior and inferior), which, along with the surface external hip rotators (gluteus maximus, posterior fibres of gluteus minimus and medius, and sartorius), act synergistically to rotate the hip [14], [17].

Up to day, tests concerning the biomechanics of dance involved the activity of selected surface external rotators of the hip joint during demi-plié [21], grand-plié [20], pirouette turn [27], relevé on demipointe [19] or grand battement devant [15]. In turn, Gilbert et al. [7] were concerned with the identification of the correlation between the hip external rotation and the functional TO in relation to five classical ballet positions. Among available subject-related scientific publications, there were no works containing the analysis of electromyography and kinematics concerning all five classical positions.

The sequence in which classical positions are taught is consistent with their numbering [22], yet, until today, it has not been verified for consistency with the level of difficulty, which is crucial in terms of the educational method of the aforesaid dancers and sports people.

The study aimed to determine which of the five classical ballet positions is the most demanding for the dancer's body. It was decided that the most demanding position would be the one in which despite of greater activity of examined muscles there would be lower external rotation of the hips and feet (foot progression angle) and there would be greater anterior pelvic tilt. We hypothesized that position 4 would appear to be the most demanding in terms of biomechanics.

Considering the most crucial elements involved in the classical ballet positions, our analysis was based on the registration of the 14 selected muscles: lumbar erector spinae, rectus abdominis, gluteus maximus, sartorius, adductor longus, rectus femoris, vastus lateralis, vastus medialis, long head of biceps femoris, semitendinosus, tibialis anterior, fibularis longus, medial and lateral heads of gastrocnemius.

\section{Materials and methods}

The cross-sectional study presented in the article aimed to identify the bioelectric activity of the mus- cles of the right side of the trunk and those of the right lower limb in five classical ballet positions adopted by pre-professional ballet dancers. Kinematic data were collected using a motion capture system.

\subsection{Subjects}

Fourteen female pre-professional ballet dancers aged 11-16 (body mass: $45.5 \pm 1.4 \mathrm{~kg}$, body height: $1.59 \pm 0.08 \mathrm{~m}$, BMI: $17.9 \pm 1.8 \mathrm{~kg} / \mathrm{m}^{2}$, experience: $4.9 \pm 1.3$ years) participated in this study (mean \pm standard deviation). All of the participants attended the same ballet school and were tutored by the same teacher in classical dance classes. Each dancer was in good health and had not suffered any injuries in the past 6 months. All of the test participants and their parents were informed about the purpose of the study and agreed (in writing) to participate in the test before the commencement of the experiment. The experiment was approved by the Bioethical Committee of the Poznan University of Medical Sciences.

\subsection{Data collection}

The test participants were tasked with the sequential adoption of five classical ballet positions, i.e., first (CP1) and second (CP2) (symmetric) as well as third (CP3), fourth (CP4) and fifth (CP5) (asymmetric with the right lower extremity forward [forward setting] and backward [backward setting]). The above-named classical positions are presented in Fig. 1. Before CP1-CP5, each dancer made the sixth classical ballet position (CP6), which was the reference position, i.e., the standing upright position with feet positioned in parallel at the width of the hips. All of the dancers performed 3 trials of each position in the following sequence $\mathrm{CP} 6, \mathrm{CP} 1, \mathrm{CP} 2, \mathrm{CP} 3$ forward, CP3 backward, CP4 forward, CP4 backward, CP5 forward and CP5 backward (a total of 27 trials). The experiment included measurements involving the entire group, first in the reference position and next in positions CP1, CP2, CP3, CP4 up to CP5. During each trial, the participant stood barefoot for 30 seconds. The interval between subsequent trials lasted approximately 1 minute, whereas the interval between individual positions lasted approximately 20 minutes. The correctness of classical positions was monitored by a teacher who had been a principal dancer at the Poznan Grand Theatre. The test participants were requested to stand in classical positions "as at an exam". 
The activity of selected muscles was recorded using a Telemyo 2400T G2 device (Noraxon, USA) compatible with the MyoResearch XP Master Edition software. The tests involved the use of surface electrodes. The EMG signal was sampled at a frequency of $1000 \mathrm{~Hz}$ and, next, filtered (bandwidth: $10-500 \mathrm{~Hz}$ ). The test participants were prepared for the test in accordance with the SENIAM guidelines [13]. Before the attachment of electrodes, the skin was shaven and cleaned with an abrasive and disinfecting agent. A pair of $\mathrm{Ag} / \mathrm{AgCl}$ electrodes (SORIMEX, Poland, $1 \mathrm{~cm}$ in diameter) was attached in the bipolar configuration along the longitudinal axis of the examined muscles of the right side of the trunk and the right lower limb: lumbar erector spinae (ES), rectus abdominis (RA),

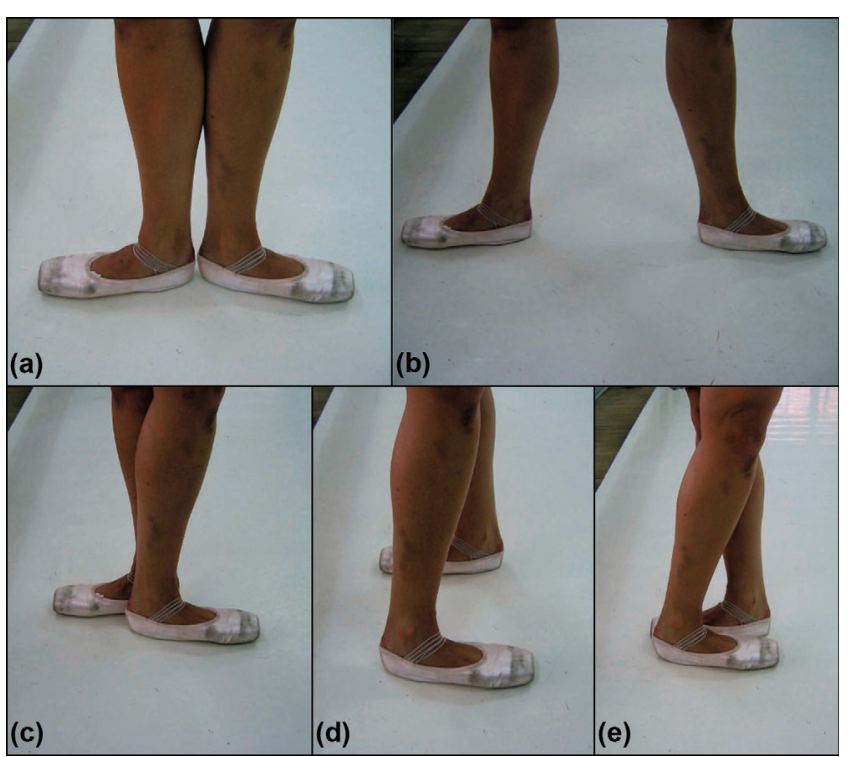

Fig. 1. Feet setting for the (a) first classical position (CP1), (b) second classical position (CP2), (c) third classical position (CP3), (d) fourth classical position (CP4) and (e) fifth classical position (CP5)

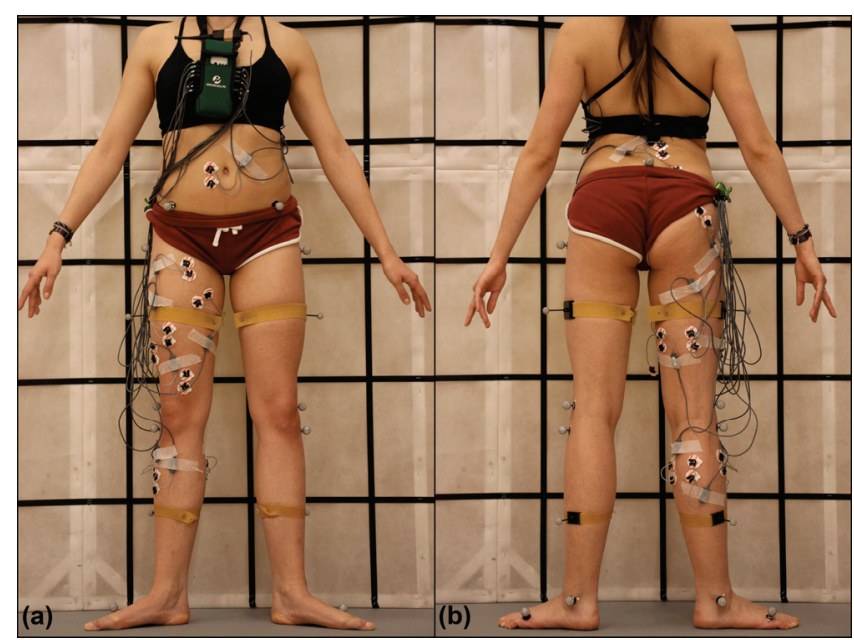

Fig. 2. Electrode placement for the classical ballet position: (a) front view, (b) back view gluteus maximus (GM), sartorius (SAR), adductor longus (ADD), rectus femoris (RF), vastus lateralis (VL), vastus medialis $(\mathrm{VM})$, long head of biceps femoris $(\mathrm{BF})$, semitendinosus (SEM), tibialis anterior (TA), fibularis longus (FIB), medial and lateral heads of gastrocnemius (MGAS and LGAS, respectively) (Fig. 2). The distance between the electrodes amounted to $2 \mathrm{~cm}$. The correctness of the attachments was verified through the observation of a raw EMG signal during the testing of the muscles. The ground electrode was placed over the right posterior superior iliac spine. All of the electrodes were attached to the test participants by the same operator.

Three-dimensional data related to the angles of individual joints of the lower limbs were collected using the BTS Smart D optoelectronic motion capture system (BTS Bioengineering, Milan, Italy) interacting with the BTS Smart Capture software programme. Assessment included the hip joint external rotation (HER), knee joint external rotation (KER), foot progression angle (FPA) and the pelvis position (tilt) in the sagittal plane (PTILT). Tests were recorded using 8 digital infrared cameras with a recording frequency of $200 \mathrm{~Hz}$. A total of 19 reflective markers (diameter: $20 \mathrm{~mm}$ ) were placed using double-sided adhesive tape on specific anatomic landmarks: right and left anterior superior iliac spines, sacrum between posterior superior iliac spines, femoral greater trochanter, marker on the bar on the lateral side of the thigh, femoral lateral epicondyle, head of fibula, marker on the bar on the lateral side of the shank, lateral malleolus, calcaneus and head of the fifth metatarsal (Fig. 2). The attachment of all markers were according to Vaughan-Davis model [6], [23] and performed by the same investigator.

\subsection{Data analysis}

The EMG signal was processed using the MyoResearch XP Master Edition software program (Noraxon, USA). Related artefacts and noise were inspected visually. The EMG signal was full-wave rectified and smoothed using the root mean square algorithm with a 50-milisecond window.

The EMG signal recorded during 3 trials was averaged in relation to all test participants and each test position. To enable the comparison of amplitude values between test participants and between muscles, the EMG signal obtained in CP6 was treated as a reference value. The EMG signal recorded in CP1-CP5 was normalized to $\mathrm{EMG}_{\mathrm{CP} 6}$ according to the following formula:

$$
\mathrm{EMG}_{\mathrm{CP} n \text { normalized }}=\mathrm{EMG}_{\mathrm{CP} n} / \mathrm{EMG}_{\mathrm{CP} 6},
$$

where $n$ is the number of each of CP1-CP5. 
Higher values translate into a higher activity of a given muscle in CP1-CP5 in comparison with the reference position.

Related kinematic data were prepared using the BTS Smart Tracker and the BTS Smart Analyzer software programs. The value of external rotation in the hip joints and knee joints, the value of pelvic anterior tilt and the foot progression angle recorded during 3 trials were averaged for all test participants and all tested positions. The algorithm of body segment rotation was based on the determination of the Euler angles, as described in [6]. The angles of rotation of both hips described the position of the thigh in relation to the pelvis. The knee rotation angle described the position of the tibia in relation to the thigh. The FPA was the absolute angle referred to the laboratory coordinate system indicating the position of the foot of a test participant in relation to the direction of progression. The pelvic plane was defined using three markers (two on anterior superior iliac spines and one at level S2 between posterior superior iliac spines). The above-named three points provided the basis enabling the calculation of the local pelvis-related coordinated system. The pelvic anterior tilt (angle PTILT) was calculated as the Euler angle between the local pelvis reference system and the global reference system located in the motion analysis laboratory [6]. In order to assess the external rotation connected with TO, the value of external rotation obtained in reference position CP6 was subtracted from that obtained in CP1-CP5.

\subsection{Statistical analysis}

The normal distribution of data was confirmed using the Shapiro-Wilk test. The study also involved the performance of repeated measures analysis of variance (ANOVA) with 2 factors for bioelectric activity (setting [forward or backward] $\times$ classical position [CP1, CP2, CP3, CP4 or CP5]) and with 3 factors for angles (LE [right or left] $\times$ setting [forward or backward] $\times$ classical position $[\mathrm{CP} 1, \mathrm{CP} 2, \mathrm{CP} 3, \mathrm{CP} 4$ or CP5]). Differences between within-subject factors were identified using the post-hoc Bonferroni test. Sphericity was verified using the Mauchly test. The Geisser-Green house adjustment was used in cases where sphericity was not obtained. The repeatability of variables from 3 trials was tested using the intraclass correlation coefficient (ICC) (95\% confidence interval). The results were subjected to statistical analysis in the SPSS software for Windows (version 25.0; IBM Corp, Armonk, NY, USA). The alpha level was set at $p \leq 0.5$ in relation to all trials.

\section{Results}

The mean (SD) standardised EMG and the kinematics exclusively for the right LE are presented in Table 1. $P$-values for comparisons related to the five classical positions are presented in Table 2 and Table 3. Table 4 shows $p$-values for comparisons between the forward and backward settings.

\subsection{EMG}

The ICC related to the bioelectric activity of 14 muscles were restricted within the range of 0.82 to 0.99 $(p<0.01)$. The effect analysis revealed significant differences in the values of bioelectric activity for the setting (ES, GM, SAR, BF, SEM, RF, LGAS, MGAS, TA) and the classical position (ES, RA, SAR, BF, SEM, ADD, RF, VLO, VMO, LGAS, MGAS, TA, FIB) $(p<$ $0.05)$. In addition, the analysis revealed a significant effect of interaction between the setting and the classical position in relation to $\mathrm{ES}, \mathrm{GM}, \mathrm{SAR}, \mathrm{BF}, \mathrm{SEM}, \mathrm{ADD}$, RF, VLO, VMO, LGAS, MGAS and TA $(p<0.05)$. Related comparisons revealed significantly higher values of activity in CP1 than those in CP2 (ES) and CP2 than CP1 (LGAS) $(p<0.05)$. Taking the forward setting into consideration, significantly higher values of activity were determined in (1) CP1 than CP3 (ES), CP4 (GM) and $\mathrm{CP} 5$ (ES), (2) CP2 than CP4 (GM, MGAS), (3) CP3 than CP1 (SEM, ADD, VLO, VMO, TA), CP2 (ADD, TA) and CP4 (GM), (4) CP4 than CP1 (SEM, ADD), CP2 (ADD, TA), CP3 (SAR, ADD) and CP5 (SAR), (5) CP5 than CP1 (SEM, ADD, VMO, LGAS, TA), CP2 (ADD, VMO, LGAS and TA), CP3 (LGAS, FIB) and CP4 (GM, LGAS, MGAS, TA and FIB) $(p<0.05)$.

Regarding the backward setting, the comparisons revealed significantly higher values of activity in (1) CP1 than CP3 (LGAS, MGAS), CP4 (LGAS, MGAS) and CP5 (BF, LGAS and MGAS), (2) CP2 than CP3 (LGAS, MGAS), CP4 (LGAS, MGAS) and CP5 (BF, SEM, LGAS, MGAS), (3) CP3 than CP1 (SAR, RF) and CP2 (ES, GM), (4) CP4 than CP1 (BF, SEM), CP2 (ES, GM), CP3 (BF, SEM) and CP5 (BF, SEM), (5) CP5 than CP1 (SAR, ADD, RF, FIB), CP2 (GM, SAR, ADD, RF), CP3 (FIB) and CP4 (SAR, RF, FIB) $(p<0.05)$. In addition, the analysis revealed significantly higher values of activity for (1) forward setting than backward setting in CP3 (BF, SEM, LGAS, MGAS, TA), CP4 (ADD, LGAS, MGAS) and CP5 (BF, SEM, VMO, LGAS, MGAS, TA) and (2) backward setting than forward setting in CP3 (ES, GM, SAR, RF), CP4 (ES, GM) and CP5 (ES, GM, SAR, RF) $(p<0.05)$. 


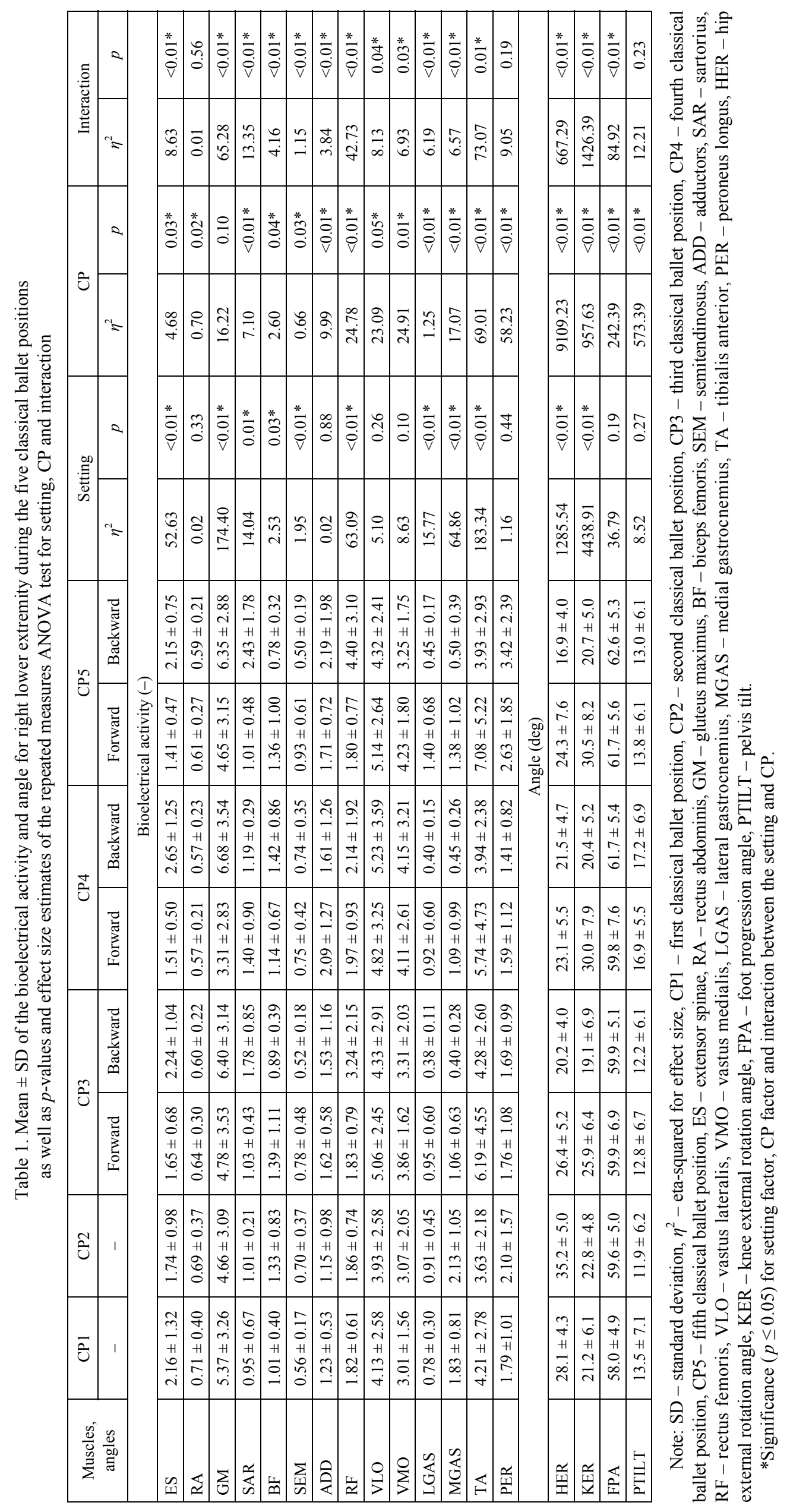




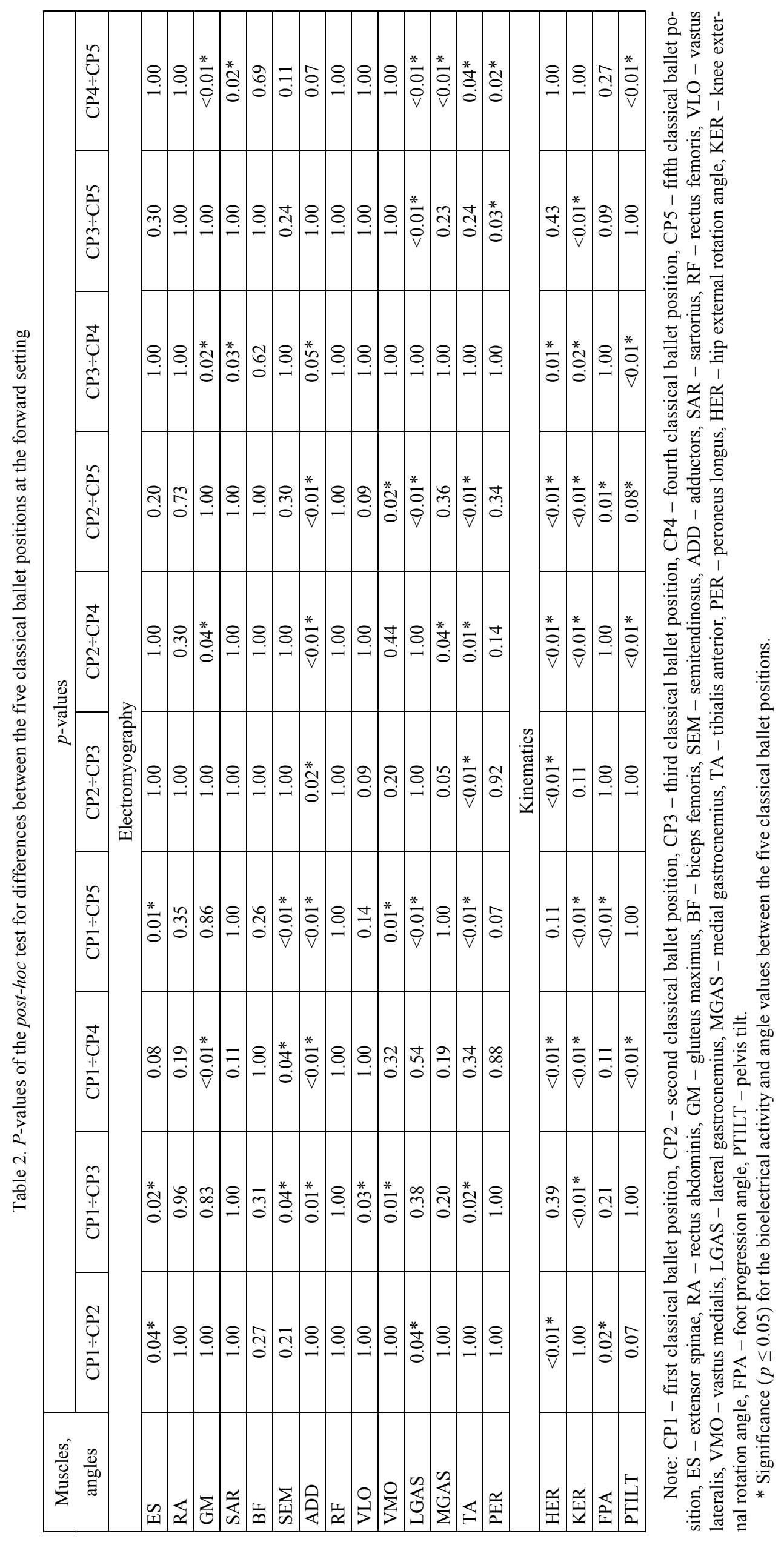




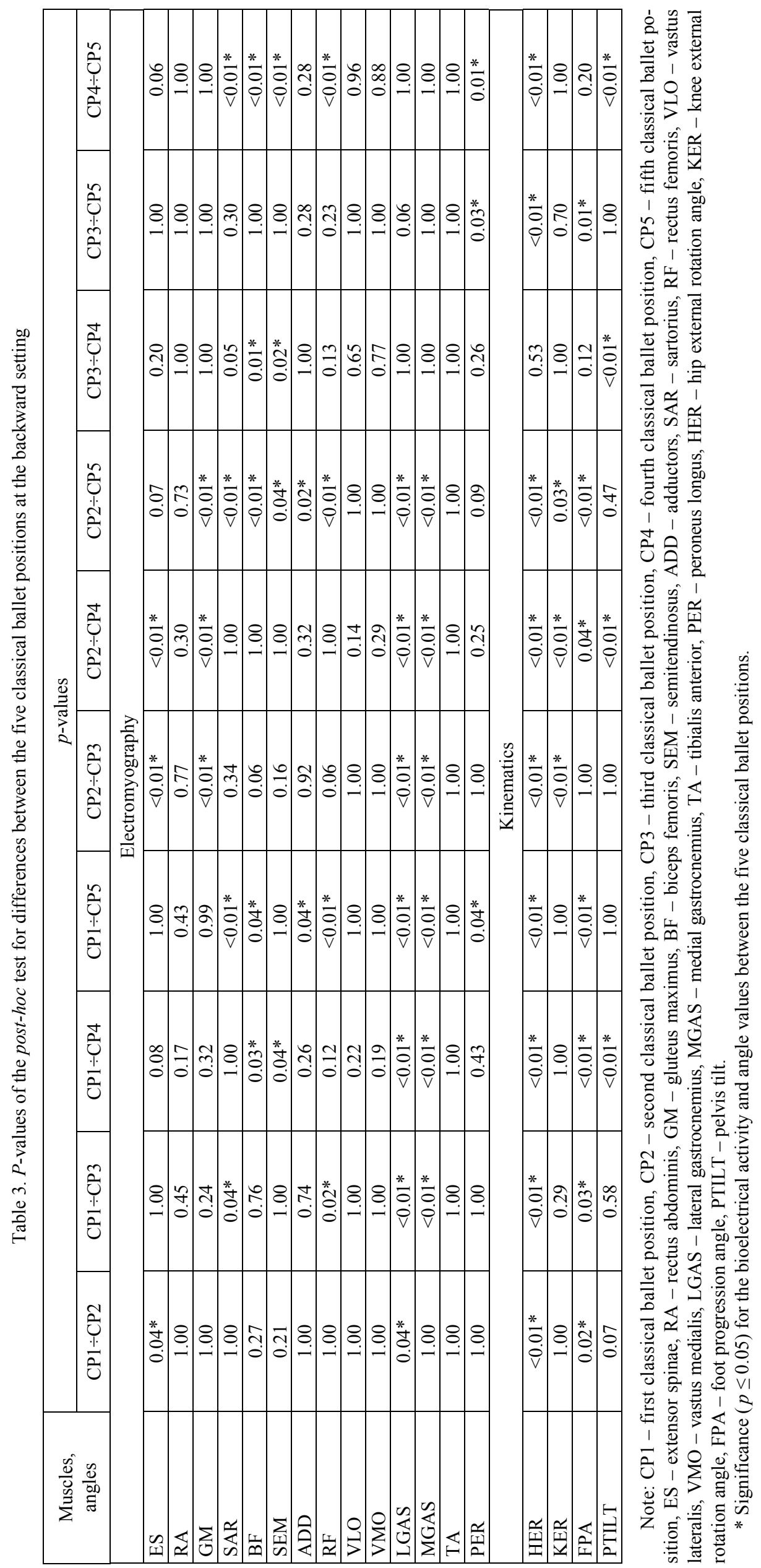


Table 4. $P$-values of the post-hoc test for differences between the forward and backward settings

\begin{tabular}{|l|c|c|c|}
\hline \multirow{2}{*}{$\begin{array}{c}\text { Muscles, } \\
\text { angles }\end{array}$} & \multicolumn{3}{|c|}{$p$-values } \\
\cline { 2 - 4 } & CP3 & CP4 & CP5 \\
\hline ES & $<0.01^{*}$ & $<0.01^{*}$ & $<0.01^{*}$ \\
\hline RA & 0.20 & 0.73 & 0.56 \\
\hline GM & $<0.01^{*}$ & $<0.01^{*}$ & $<0.01^{*}$ \\
\hline SAR & $0.02^{*}$ & 0.45 & $<0.01^{*}$ \\
\hline BF & $<0.01^{*}$ & 0.09 & $<0.01^{*}$ \\
\hline SEM & $<0.01^{*}$ & 0.80 & $<0.01^{*}$ \\
\hline ADD & 0.61 & $0.02^{*}$ & 0.09 \\
\hline RF & $<0.01^{*}$ & 0.61 & $<0.01^{*}$ \\
\hline VLO & 0.08 & 0.40 & 0.07 \\
\hline VMO & 0.08 & 0.93 & $0.01^{*}$ \\
\hline LGAS & $<0.01^{*}$ & $<0.01^{*}$ & $<0.01^{*}$ \\
\hline MGAS & $<0.01^{*}$ & $<0.01^{*}$ & $<0.01^{*}$ \\
\hline TA & $<0.01^{*}$ & 0.05 & $<0.01^{*}$ \\
\hline PER & 0.71 & 0.40 & 0.20 \\
\hline \multicolumn{5}{|c|}{ Kinematics } \\
\hline HER & $<0.01^{*}$ & 0.13 & $<0.01^{*}$ \\
\hline KER & $<0.01^{*}$ & $<0.01^{*}$ & $<0.01^{*}$ \\
\hline FPA & 0.94 & 0.11 & 0.25 \\
\hline PTILT & 0.33 & 0.57 & 0.06 \\
\hline
\end{tabular}

Note: CP3 - third classical ballet position; CP4 - fourth classical ballet position; CP5 - fifth classical ballet position; ES - extensor spinae; RA - rectus abdominis; GM - gluteus maximus; $\mathrm{BF}$ - biceps femoris; SEM - semitendinosus; ADD - adductors; SAR - sartorius; RF - rectus femoris; VLO - vastus lateralis; VMO - vastus medialis; LGAS - lateral gastrocnemius; MGAS - medial gastrocnemius; TA - tibialis anterior; PER - peroneus longus; HER - hip external rotation angle; KER - knee external rotation angle; FPA - foot progression angle; PTILT - pelvis tilt.

* Significance $(p \leq 0.05)$ for the bioelectrical activity and angle values between the forward and backward settings.

\subsection{Kinematics}

The ICC related to KER, HER, FPA and PTILT was restricted within the range of $0.88-0.99(p<0.01)$. The analysis of effects revealed significant differences in angle values in relation to the setting (HER and KER) and in relation to the classical position (HER, KER, FPA and PTILT) $(p<0.001)$. In addition, the analysis showed the significant effect of interaction between the setting and the classical position in relation to HER, KER and FPA $(p<0.01)$. Related comparisons revealed significantly higher angle values for CP2 than CP1 (HER, FPA) $(p<0.05)$. In relation to the forward setting, the comparison revealed significantly higher angle values in (1) CP1 than CP4 (HER),
(2) CP2 than CP3 (HER), CP4 (HER) and CP5 (HER), (3) CP3 than CP1 (KER), (4) CP4 than CP1 (KER, PTILT), CP2 (KER, PTILT), CP3 (KER, PTILT) and CP5 (PTILT), (5) CP5 than CP1 (KER, FPA), CP2 (KER, FPA, PTILT) and CP3 (KER) $(p<0.05)$. In relation to the backward setting, the comparison showed significantly higher angle values in (1) CP1 than CP3 (HER), CP4 (HER) and CP5 (HER), (2) CP2 than CP3 (HER, KER), CP4 (HER, KER) and CP5 (HER, KER), (3) CP3 than CP1 (FPA) and CP5 (FPA), (4) CP4 than CP1 (FPA, PTILT), CP2 (FPA, PTILT), CP3 (PTILT) and CP5 (HER, PTILT), (5) CP5 than CP1 (FPA), CP2 (FPA) and CP3 (FPA) $(p<0.05)$. The analysis also revealed significantly higher angle values only for forward setting rather than backward setting in CP3 (HER, KER), CP4 (KER) and CP5 (HER, KER) $(p<0.001)$. However, comparisons in pairs showed insignificant differences in values of all angles between the right LE (forward setting) and left LE (backward setting) as well as between the right LE (backward setting) and left LE (forward setting).

\section{Discussion}

This work aimed to identify the most demanding classical ballet position in terms of biomechanics. Because of the fact that the sequence of teaching classical positions is consistent with their numbering, it is necessary to verify the difficulty of each position and refer it to the educational methodology of dancers and sportspeople mentioned at the beginning of this article. The study enabled the identification of the activity of surface muscles in TO and the analysis of the values of the lower limb joint angles (HER, KER), setting of the foot in relation to the ground (FPA) and the pelvic inclination (PTILT) in the five classical ballet positions.

The locomotor system of ballet dancers is subjected to loads in a very peculiar way. This specific nature is concerned with the skeletal system [10] exposed to the non-uniform effect of impact forces reaching as many as $8-9$ times body weight [9], where highly-mineralised are elements directly exposed to the impact. Also, according to the previous study results obtained by Gorwa et al. [8], the muscular system must meet the technique-related requirements of classical dance where the value of HER determines the use of specific muscles.

The first year of classical dance education sees the beginning of work aimed to master each if the five classical ballet positions to be followed by the intro- 
duction of plié ("squat") for these positions. Initially, children are taught demi-plié (half-squat) and next, grand-plié (full squat). This requires a complicated coordination of dynamic muscular activities (when making plié) and static muscular activities (when stabilising the trunk and lower limbs) [12].

These two components of dancer's training, i.e., classical ballet positions and plié belong to the most frequently performed moves [12]. The base of plié includes classical positions. It is possible to make plié or demi-plié starting from each of the five ballet positions. Apart from a few exceptions, plié precedes and ends most steps in the classical dance technique and choreography. In plié, muscular activity lies in eccentric contraction [25]. The sequence used when teaching classical ballet positions to children is consistent with their naming, yet their technical difficulty, according to certain teachers, does not overlap with the aforesaid naming. According to an opinion by a multi-annual classical dance teacher, the grading of difficulty (the most to the least demanding) is the following: CP4, CP5, $\mathrm{CP} 2, \mathrm{CP} 3$ and $\mathrm{CP} 1$.

In terms of biomechanics and from a perspective of proper execution of classical ballet positions, static muscular activity dominates during the training. Isometric contraction constitutes a significant percentage of the muscular activity of ballet dancers [12]. The aforesaid muscular activity results from the necessity of stabilising the trunk and pelvis. According to Haight [12], the greatest "back-up" for ballet includes strong lower limbs and strong muscles of the trunk. The biomechanical analysis of the human locomotor system revealed that unfavourable situations are those when muscles are subjected to long isometric tension and when muscles stabilise the multisegment biomechanism bases. It seems that the five classical ballet positions fall ideally within the scheme of the aforesaid static loads.

Taking the values of muscular activities and those of related angles into consideration, the following correlations in individual positions were observed.

\subsection{Symmetric positions: the first and second ballet position}

Vaganova [22] defines the first and second position as follows: "In the first position, the balls of the both feet are completely turned out, the heels touch and the feet form a single straight line. In the second position, the balls of both feet are so one line, but there is a distance of one foot between the heels".

Ballet teachers instruct students how to maintain the ideal $\mathrm{CP} 1$ by giving them the following principles: (1) buttocks must be tightened to keep the HER, (2) feet must be supinated so that their lateral border will be depressed and closely adhere to the floor while their medial arch is elevated, (3) toes must be relaxed and placed on the floor, (4) the center of the patella should be above the second toe [16], (5) hip joints must not be flexed but held in neutral anatomical position, (6) thighs should be held together (only in the CP1), (7) abdominal and back muscles must be tightened and form the foundation for the HER [8].

The technically correct $\mathrm{CP} 1$ requires the activity of the (1) deep and superficial hip external rotators [15], [17], (2) trunk muscles, essential for keeping stable straight posture [3], [17], [24], (3) ankle muscles that act antagonistically to pronate or supinate and dorsior plantarflex the foot. The EMG tests revealed that symmetric positions $\mathrm{CP} 1$ and $\mathrm{CP} 2$ weigh down the muscular system in a similar manner. A significant difference is a higher activity of back muscles (ES) and a lower activity of foot muscles (LGAS) in CP2 than in CP1.

In terms of biomechanics, $\mathrm{CP} 2$ is characterised by higher stance plane enabling the dancer to maintain higher postural stability. In turn, in CP1, the static muscular activity of lower limbs (equilibrium of force moments) is balanced by the possibility of touching one's feet. Interestingly, in spite of significant similarity in terms of technique, considerable differences regarding the aforesaid positions in HER and FPA can be noticed. The CP2 generates higher values of the aforementioned angles. In addition, in CP2 HER reaches its highest value $\left(35.2^{\circ}\right)$ in comparison with other classical ballet positions.

\subsection{Asymmetric positions: the third, fourth and fifth classical ballet position}

According to Vaganova [22], proper asymmetric positions are determined by the following technical details: „In the third position, one foot is in front of the other, heels touching the middle of the other foot. The fourth position is similar to the third, the feet being parallel and one short step apart. In the fifth position, both feet touch so that the toe of one foot reaches the heel of the other". 
Instructions provided by the classical dance teacher to children and concerned with asymmetric positions sound the same as those referring to symmetric positions except for instructions related to the upper part of the body: "think intensively about the pelvis, abdomen and buttocks, yet let your iliac spine look forward, and the hip rotates in the acetabulum".

In terms of maintaining TO there is no ideal asymmetric position (CP3-CP5) and the possibility of execution of the above-named positions both with the right lower limb forward (forward setting) or with the right lower limb backward (backward setting) makes the aforesaid positions extremely difficult to master [8]. Taking various manners in which classical ballet positions can be performed into consideration, this study contains the analysis of separate, i.e., forward and backward, settings of lower limbs in relation to asymmetric positions.

In the asymmetric positions, the pelvis is rotated towards the lower limb in the back, which naturally increases the external rotation in the hip of the lower limb in the front (which was confirmed in the present study). In relation to the forward setting, it was possible to observe a higher activity of muscles "supporting" the foot. As a result, using appropriate movements of the foot, dancers try to correct the oblique position of the pelvis, which is rotated towards the lower limb in the back. In the forward setting, it is more difficult to position the foot perpendicular to the axis in the sagittal plane. The foregoing (according to presented study results) imposes greater activation of LGAS, MGAS and TA. This difficulty appeared only in the EMG tests. There were no differences in FPA between the forward and backward settings. Until today, information contained in subject-related reference publications was concerned with the goniometric or kinematic assessment of classical ballet positions. As it turned out, a complete evaluation of their difficulty and correctness requires the inclusion of the EMG-based assessment.

In relation to the backward setting, the activity of muscles externally rotating the hip (GM, SAR) was higher than that in the forward setting. Because of the fact that the pelvis is rotated towards the lower limb in the back, dancers try to rotate the pelvis so that it reaches a neutral position (in accordance with the teacher's instruction) in the frontal plane and are "forced" to activate the external rotators of the hip on the backward side. In spite of the increased muscular activity of GM and SAR on the backward side, external rotation in the hip joint was greater in the forward setting than that in the backward setting in positions $\mathrm{CP} 3$ and $\mathrm{CP} 5$. In addition, asymmetric positions in the backward setting required a lower activity of the mus- cles supporting the foot (LGAS and MGAS) in comparison with that observed in symmetric positions. At the same time, in asymmetric positions, the dancers achieved higher FPA in the backward setting than in symmetric positions. The aforesaid observations confirmed that dancers do not experience difficulty positing the foot in the external rotation in the backward setting.

In $\mathrm{CP}$, muscular activation was characteristic for asymmetric positions. The forward setting was responsible for a greater activity of the muscles supporting the foot (TA), whereas the backward setting to a greater extent activated the muscles supporting the hip joint (GM, SAR). CP3 was characterised by lower activation of the muscles supporting the foot (LGAS, MGAS) than in cases of symmetric positions CP1 and $\mathrm{CP} 2$. In addition, in comparison with the two remaining asymmetric positions CP4 and CP5, CP3 did not trigger a greater activity of muscular groups supporting the hip joint or the foot.

CP4 is the most demanding position to learn because of the difficulty in positioning the hips in one line and the stabilisation of the feet. CP4 constitutes the base for many elements of the classical dance as well as for "ordinary" walking on the stage or for a technical element known as "échappé" [22]. In CP4, in the forward setting, the activity of GM was lower than in the other positions. In addition, the values of the external rotation of the hip in $\mathrm{CP} 4$ forward were lower than those in $\mathrm{CP} 1$ and $\mathrm{CP} 2$. Also, in position CP4 in both settings, the pelvic anterior tilt was greater than in the other positions. The aforesaid results indicate greater difficulty in maintaining CP4 than other classical ballet positions. The pelvic anterior tilt triggers relative flexion in the hip joint, which relaxes the ligamentous apparatus within the hip joint [26] but, at the same time, increases the lumbar lordosis [11]. In turn, the hyperlordosis is connected with the low back pain which professional dancers suffer from.

CP5 forward entailed a higher activity of muscles supporting the foot (primarily LGAS, but also MGAS, TA and FIB) than in the remaining positions. The foregoing implies that the most demanding task in CP5 is the maintaining of the foot in the forward setting. In general, the forward setting in asymmetric positions may impede the rotation of the foot so that its longitudinal axis is perpendicular to the axis on the sagittal plane. However, in terms of the FPA, the results for CP5 forward are slightly lower than those for other asymmetric positions and higher in comparison with the results obtained for both symmetric positions. 


\section{Conclusions}

The use of two measurement systems including the EMG and motion capture enabled the full assessment of the difficulty of the five classical ballet positions. This study is the first such analysis of all five classical ballet positions.

The most demanding positions in the classical dance, for the dancer's body, are CP4 and CP5. This study demonstrated that $\mathrm{CP} 4$ is the most demanding of all of the five ballet positions. CP4 is characterised by the greatest pelvic anterior tilt in both forward and backward settings as well as by a lower activity of GM in the forward setting in comparison with the remaining positions.

The test results also revealed that dancers find it more demanding to perform a proper TO in relation to the forward setting than backward setting in CP3, CP4 and CP5. In asymmetric positions, it is the lower limb forward that "experiences" the greatest difficulty. In addition, in asymmetric positions, dancers experience difficulties connected with the necessity of maintaining the proper $\mathrm{TO}$ at the level of hips, with their lower limbs crossed.

Symmetric positions are not as demanding as asymmetric. Higher values of HER and FPA in CP2 indicate that this position is easier than CP1 (the dancers performed the turnout without more intense muscular activity). Additionally, CP1 is characterised by an increased activity of back muscles.

These findings can be applied in educational method of dancers. It is suggested that the sequence of teaching classical ballet positions should rely on their difficulty level, not their numbering. Based on the present study results, the grading of difficulty from the most to the least demanding classical ballet position and therefore suggested sequence of teaching is the following: CP2, CP1, CP3, CP5 and CP4.

\section{Acknowledgements}

The authors would like to thank all participating female dancers and their parents for consent as well as Mrs Apolonia Myszkowska for supervising the technical correctness of studied positions. This work was supported by the Polish Ministry of Science and Higher Education under Grant NN404515938.

\section{References}

[1] Bogdanova M., Regarding the methods of the preparation of the ballroom dancing performers, National Academy of Managerial Staff of Culture and Arts Herald, 2015, 4, 65-69, DOI: 10.32461/2226-3209.4.2015.138430.
[2] Bolia I., Utsunomiya H., Locks R., BriggS K., PHILIPPON M., Twenty-year systematic review of the hip pathology, risk factors, treatment, and clinical outcomes in artistic athletes-dancers, figure skaters, and gymnasts, Clin. J. Sport Med., 2018, 28 (1), 82-90, DOI: 10.1097/ JSM.0000000000000440.

[3] Champion L.M., Chatfield S.J., Measurement of turnout in dance research. A critical review, J. Dance Med. Sci., 2008, 12 (4), 121-135.

[4] Coplan J.A., Ballet dancer's turnout and its relationship to self-reported injury, J. Orthop. Sports Phys., 2002, 32 (11), 579-584, DOI: 10.2519/jospt.2002.32.11.579.

[5] Danardani W., Soegiyanto K., Setijono H., Sulaiman S., Artistic swimming helps the intelligence development. Proceedings of the international seminar on public health and education 2018 (ISPHE 2018), Adv. Health Sci. Res., 2018, 12, 81-85, DOI: 10.2991/isphe-18.2018.18.

[6] Davis R.B., OunPuU S., Tyburski D., GaGe J.R., A gait analysis data collection and reduction technique, Hum. Mov. Sci., 1991, 10 (5), 575-587, DOI: 10.1016/0167-9457(91)90046-Z.

[7] Gilbert C.B., Gross M.T., Klug K.B., Relationship between hip external rotation and turnout angle for the five classical ballet positions, J. Orthop. Sports Phys. Ther., 1998, 27 (5), 339-348, DOI: 10.2519/jospt.1998.27.5.339.

[8] Gorwa J., KabacińsKi J., Murawa M., FrYZOwicz A., On the track of the ideal turnout: Electromyographic and kinematic analysis of the five classical ballet positions, PLoS ONE, 2020, 15 (3), e0230654, DOI: 10.1371/journal.pone.0230654.

[9] Gorwa J., Michnik R., Nowakowska K., JuRKoJĆ J., JOCHYMCZYK-WOŹNIAK K., Is it possible to reduce loads of the locomotor system during the landing phase of dance figures? Biomechanical analysis of the landing phase in Grand Jeté, Entrelacé and Ballonné, Acta Bioeng. Biomech., 2019, 21 (4), 111-121, DOI: 10.5277/ABB-01429-2019-02.

[10] GORWA J., ZIELIŃSKI J., WOLAŃSKI W., MiCHNIK R., LARYSZ, D., DwORAK L.B., KusY K., Decreased bone mineral density in forearm vs loaded skeletal sites in professional ballet dancers, Med. Probl. Perform. Art., 2019, 34 (1), 25-32, DOI: 10.21091/mppa.2019.1006

[11] GotTschlich L.M., Young C.C., Spine injuries in dancers, Curr. Sports Med. Rep., 2011, 10 (1), 40-44, DOI: 10.1249/ JSR.0b013e $318205 \mathrm{e} 08 \mathrm{~b}$.

[12] HAIGHT H.J., Morphologic, physiologic, and functional interactions in elite female ballet dancers, Med. Probl. Perform. Art., 1998, 13 (1), 4-13.

[13] Hermens H.J., Freriks B., Disselhorst-Klug C., Rau G., Development of recommendations for SEMG sensors and sensor placement procedures, J. Electromyogr. Kinesiol., 2000, 10 (5), 361-374, DOI: 10.1016/s1050-6411(00)00027-4.

[14] Howse J., McCormack M., Anatomy, dance technique and injury prevention, A \& C Black Publishers Ltd., London, England, 2009.

[15] Krasnow D., Ambegaonkar J.P., Wilmerding M.V., STECYK S., Koutedakis Y., WyON M., Electromyographic comparison of grand battement devant at the barre, in the center, and traveling, Med. Probl. Perform. Art., 2012, 27 (3), 143-155, DOI: 10.1016/s1050-6411(00)00027-4.

[16] QuANBECK A.E., RusSELl J.A., HANDLEY S.C., QuANBECK D.S., Kinematic analysis of hip and knee rotation and other contributors to ballet turnout, J. Sports Sci., 2017, 35 (4), 331-338, DOI: 10.1080/02640414.2016.1164335.

[17] Sherman A.J., Mayall E., TASKer S.L., Can a prescribed turnout conditioning program reduce the differential between 
passive and active turnout in pre-professional dancers? J. Dance Med. Sci., 2014, 18 (4), 159-168, DOI: 10.12678/ 1089-313X.18.4.159.

[18] Skwiot M., Śliwiński G., Milanese S., ŚliwiŃSki Z., Hypermobility of joints in dancers, 2019, PLoS ONE, 14 (2), e0212188, DOI: 10.1371/journal.pone.0212188.

[19] Tanabe H., Fujir K., KouzaKi M., Joint coordination and muscle activities of ballet dancers during tiptoe standing, Motor Control, 2017, 21 (1), 72-89, DOI: 10.1123/ mc.2015-0002.

[20] Trepman E., Gellman R.E., Micheli L.J., De Luca C.J., Electromyographic analysis of grand-plié in ballet and modern dancers, Med. Sci. Sports Exerc., 1998, 30 (12), 1708-1720, DOI: 10.1097/00005768-199812000-00009.

[21] Trepman E., Gellman R.E., Solomon R., Murthy K.R., Micheli L.J., DE LuCA C.J., Electromyographic analysis of standing posture and demi-plié in ballet and modern dancers, Med. Sci. Sports Exerc., 1994, 26(6), 771-782, DOI: 10.1249/ 00005768-199406000-00018.
[22] Vaganova A., Basic principles of classical ballet. Russian ballet technique, Dover Publications, Inc., New York 2015.

[23] Vaughan C., Davis B., O'Connor J., Dynamics of human gait, Kiboho Publishers, 1992.

[24] Washington I., Mayes S., Genderton C., Pizzari T., Differentials in turnout among professional classical ballet dancers, Med. Probl. Perform. Art., 2016, 31 (3), 160-165, DOI: $10.21091 / \mathrm{mppa} .2016 .3029$.

[25] Westblad P., Tsai-Fellander L., Johansson C., Eccentric and concentric knee extensor muscle performance in professional ballet dancers, Clin. J. Sport Med., 1995, 5 (1), 48-52, DOI: 10.1097/00042752-199501000-00009.

[26] Woodhull-McNeal A.P., Clarkson P.M., James R., Watkins A., Barrett S., How linear is dancers' posture? Med. Probl. Perform. Art., 1990, 5 (4), 151-154.

[27] ZaFeriou A.M., Flashner H., Wilcox P.R., MCNitT-Gray J.L., Lower extremity control during turns initiated with and without hip external rotation. J. Biomech., 2017, 52, 130-139, DOI: 10.1016/j.jbiomech.2016.12.017. 